\title{
Musing on cell therapy for aortic aneurysms
}

Alessandro Della Corte, $\mathrm{MD}, \mathrm{PhD},{ }^{\mathrm{a}}$ Michele Di Mauro, $\mathrm{MD}, \mathrm{PhD},{ }^{\mathrm{b}}$ and Amalia Forte, $\mathrm{MD}, \mathrm{PhD}^{\mathrm{c}}$

From the Departments of a Cardiothoracic Sciences, and ${ }^{c}$ Experimental Medicine, University of Campania "L. Vanvitelli," Naples, Italy; and 'Department of Cardiovascular Disease, University of L'Aquila, L'Aquila, Italy.

Disclosures: Authors have nothing to disclose with regard to commercial support.

Received for publication Feb 7, 2018; accepted for publication Feb 11, 2018; available ahead of print March 16, 2018

Address for reprints: Michele Di Mauro, MD, PhD, Cardiovascular Disease Department, University of L'Aquila, Piazzale Tommasi 1, L’Aquila, 67100, Italy (E-mail: mdimauro1973@gmail.com).

J Thorac Cardiovasc Surg 2018;155:2314-5

$0022-5223 / \$ 36.00$

Copyright $(c) 2018$ by The American Association for Thoracic Surgery

https://doi.org/10.1016/j.jtcvs.2018.02.027

Abdominal aortic aneurysm (AAA) is a progressive disease with a high mortality and an increasing prevalence with aging. AAA formation is a multifactorial process, involving, among others, destructive remodeling of the connective tissue, mediated by smooth muscle cell changes and endothelial cell dysfunction.

Currently, the only strategy to prevent rupture and death related to AAA is surgical intervention. Several therapeutic approaches to prevent or reduce the progression of AAA have been tested so far in patients and in animal models of disease, including traditional pharmacologic approaches, such as renin-angiotensin system blockers and macrolides $^{1,2}$ and cell therapy-based strategies. ${ }^{3}$ Despite initially encouraging results, however, their ultimate outcomes of these therapies have so far been unsatisfactory.

In this context, we read with interest the study in this issue of the Journal by Hosoyama and colleagues ${ }^{4}$ summarizing the results of a cell therapy protocol tested in a murine model of AAA. The protocol is based on a relatively recently discovered subpopulation of mesenchymal stromal stem cells (MSCs), the multilineage-differentiating stressenduring (Muse) cells, identified and characterized by this same research group, led by Dezawa, ${ }^{5}$ in 2010 . Muse cells are characterized by the expression of the surface marker stage-specific embryonic antigen 3 , shared also by embryonic stem cells. Muse cells are endogenous, nontumorigenic, pluripotentlike stem cells that reside in the connective tissue of nearly every organ, as well as in the bone marrow and in peripheral blood. Muse cells are of great interest because, unlike other MSCs, they retain a pluripotent potential and are able to self-renew and to differentiate into cells representative of all 3 germ layers. Moreover, Muse cells are able to migrate and home at an injury site, regenerating the damaged tissue even in the presence of proapoptotic and proinflammatory factors.

The results obtained by Hosoyama and colleagues ${ }^{4}$ in mice highlight the remarkable homing and differentiation abilities of Muse cells in the damaged aortic wall and are of great interest from the perspective of a cell therapy-based approach able to slow down aneurysm progression or prevent

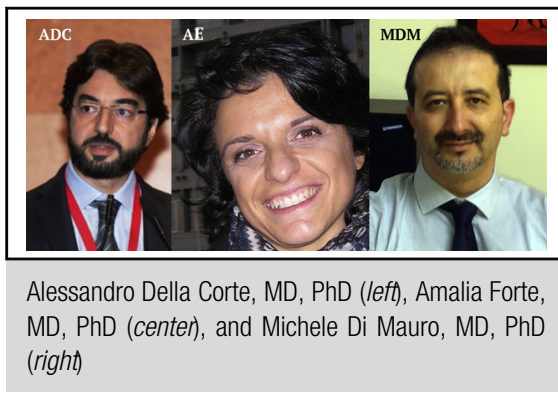

Central Message

The study by Hosoyama and colleagues confirms and extends the potential of Muse cells as a valuable tool in cell-based therapy for aortic aneurysm.

See Article page 2301.

aortic aneurysm rupture. It would be of great interest if the results that they have obtained in a model of AAA, mainly driven by atherosclerosis and inflammation, could be expanded to thoracic aortic aneurysm, which is characterized by partially different pathogenetic mechanisms. The present authors have reported that the Muse cells home at dilated aorta after migration through adventitial vasa vasorum. ${ }^{4}$ This aspect is of particular interest because the cell therapy protocol proposed by Hosoyama and colleagues ${ }^{4}$ takes advantage of the increased vasa vasorum neovascularization found in surgical specimens retrieved at the time of aneurysm repair and presumably driven by inflammation. ${ }^{6,7}$ Of note, increased adventitial vascularization has also been associated with human thoracic aortic aneurysm, ${ }^{8}$ thus expanding the potential application of the Muse-based cell therapy protocol tested in mice. Moreover, Muse cells offer the advantages of being easily accessible and of a doubling time of 1.3 days/cell division in vitro, further supporting their use in allogeneic or autologous transplant in patients.

The use of severe combined immunodeficient mice, selected to exclude the influence of host immune response as a confounding factor, could potentially have affected the homing and engraftment of administered Muse cells, because inflammation and consequent infiltration of immune cells play a key role in AAA. This consideration suggests that a repetition of the cell therapy protocol in wild type mice is necessary, as previously done for human umbilical cord MSCs, ${ }^{9}$ to verify its effectiveness in the presence of a fully efficient immune system before progressing toward a possible clinical application. The immunomodulatory properties of Muse cells mediated by the secretion of specific 
factors should support their application in wild-type models of disease and in patients. ${ }^{10}$

The differentiation abilities of the Muse cells were the main object of this study. Paracrine effects are also known as a main mechanism of action of total bone marrow MSCs,${ }^{11}$ however, so they should be evaluated in vivo in Muse cells as well.

Finally, it would be interesting to assess Muse cell homing and effectiveness also in stabilizing already developed AAA, in a later phase after aneurysm induction (the current study was limited to Muse injections at days 0,7 , and 14 after AAA induction). This test could provide indications regarding the potential application of Muse cells in a clinical setting, where aortic dilation is presumably already present at diagnosis and has been progressing for years.

In conclusion, the study by Hosoyama and colleagues ${ }^{4}$ confirms and extends the potential of Muse cells as a valuable tool in cell-based therapy of aortic aneurysm by virtue of their remarkable differentiation abilities, homing at injury sites, stress tolerance, and immunomodulatory properties. It is to be hoped that additional preclinical experiments in models of aneurysm will support the translation toward their clinical application, as has already recently happened for MSCs in patients with small AAAs. ${ }^{12}$

\section{References}

1. Salata K, Syed M, Hussain MA, Eikelboom R, de Mestral C, Verma S, et al. Renin-angiotensin system blockade does not attenuate abdominal aortic aneurysm growth, rupture rate, or perioperative mortality after elective repair. $J$ Vasc Surg. 2018;67:629-36.e2.
2. Wang YD, Liu ZJ, Ren J, Xiang MX. Pharmacological therapy of abdominal aortic aneurysm: an update. Curr Vasc Pharmacol. 2018;16:114-24.

3. Giraud A, Zeboudj L, Vandestienne M, Joffre J, Esposito B, Potteaux S, et al. Gingival fibroblasts protect against experimental abdominal aortic aneurysm development and rupture through tissue inhibitor of metalloproteinase-1 production. Cardiovasc Res. 2017;113:1364-75.

4. Hosoyama K, Wakao S, Kushida Y, Ogura F, Maeda K, Adachi O, et al. Intravenously injected human multilineage-differentiating stress-enduring cells selectively engraft into mouse aortic aneurysms and attenuate dilatation by differentiating into multiple cell types. J Thorac Cardiovasc Surg. 2018;155: 2301-13.e4.

5. Kuroda Y, Kitada M, Wakao S, Nishikawa K, Tanimura Y, Makinoshima H, et al. Unique multipotent cells in adult human mesenchymal cell populations. Proc Natl Acad Sci U S A. 2010;107:8639-43.

6. Maiellaro K, Taylor WR. The role of the adventitia in vascular inflammation. Cardiovasc Res. 2007;75:640-8.

7. Mäyränpää MI, Trosien JA, Fontaine V, Folkesson M, Kazi M, Eriksson P, et al Mast cells associate with neovessels in the media and adventitia of abdominal aortic aneurysms. J Vasc Surg. 2009;50:388-95; discussion 395-6.

8. Kessler K, Borges LF, Ho-Tin-Noé B, Jondeau G, Michel JB, Vranckx R. Angiogenesis and remodelling in human thoracic aortic aneurysms. Cardiovasc Res. 2014; 104:147-59.

9. Sharma AK, Salmon MD, Lu G, Su G, Pope NH, Smith JR, et al. Mesenchymal stem cells attenuate NADPH oxidase-dependent high mobility group box 1 production and inhibit abdominal aortic aneurysms. Arterioscler Thromb Vasc Biol. 2016;36:908-18.

10. Gimeno ML, Fuertes F, Barcala Tabarrozzi AE, Attorressi AI, Cucchiani R, Corrales L, et al. Pluripotent nontumorigenic adipose tissue-derived Muse cells have immunomodulatory capacity mediated by transforming growth factor- $\beta 1$. Stem Cells Transl Med. 2017;6:161-73.

11. Forte A, Rinaldi B, Sodano L, Berrino L, Rossi F, Finicelli M, et al. Stem cel therapy for arterial restenosis: potential parameters contributing to the success of bone marrow-derived mesenchymal stromal cells. Cardiovasc Drugs Ther. 2012;26:9-21.

12. Wang SK, Green LA, Gutwein AR, Drucker NA, Motaganahalli RL, Fajardo A, et al. Rationale and design of the ARREST trial investigating mesenchymal stem cells in the treatment of small abdominal aortic aneurysm. Ann Vasc Surg. 2018; 47:230-7. 